\title{
Corela
}

Cognition, représentation, langage

9-1 | 2011

Vol. $9, n^{\circ} 1$

\section{Représentation idéographique des prépositions russes /v/ et /na/}

Tatsiana Vavula

\section{(2) OpenEdition}

\section{Journals}

Édition électronique

URL : https://journals.openedition.org/corela/1935

DOI : $10.4000 /$ corela. 1935

ISSN : 1638-573X

Éditeur

Cercle linguistique du Centre et de I'Ouest - CerLICO

Référence électronique

Tatsiana Vavula, «Représentation idéographique des prépositions russes /v/ et /na/ », Corela [En ligne], 9-1 | 2011, mis en ligne le 06 juin 2011, consulté le 21 septembre 2021. URL : http:// journals.openedition.org/corela/1935; DOI : https://doi.org/10.4000/corela.1935

Ce document a été généré automatiquement le 21 septembre 2021

\section{(c) (i) (2)(2)}

Corela - cognition, représentation, langage est mis à disposition selon les termes de la licence Creative Commons Attribution - Pas d'Utilisation Commerciale - Partage dans les Mêmes Conditions 4.0 International. 


\title{
Représentation idéographique des prépositions russes / $/$ et / na/
}

\author{
Tatsiana Vavula
}

\section{Introduction}

1 Les prépositions russes auxquelles nous nous intéressons - B (/v/) et $\mathrm{HA}(/ \mathrm{na} /)$ - sont polysémiques. Leur identité ne se réduit pas à une étiquette ramenant leur sens à l'expression d'une seule relation d'autant que cette étiquette ne concerne pas la préposition elle-même mais l'interprétation qu'elle est susceptible de prendre selon le contexte.

2 Parmi les correspondants français de /na/ nous pourrions citer au moins 14 prépositions différentes : sur, à, dans, en, de, entre, pour, contre, par, pendant, vers, avec, après, sous. La préposition sur compte le plus grand nombre d'emplois équivalents à /na/. Par conséquent, nous la considérons en tant que correspondant principal de la préposition / $\mathrm{na} /$. Les prépositions françaises équivalentes à /v/ sont au moins 13 : dans, en, à, sur, de, pour, avec, entre, par, sous, pendant, parmi, derrière parmi lesquelles nous distinguons le premier correspondant de / $/$ / en français - la préposition dans.

3 Nous pouvons toujours sélectionner un « équivalent principal » pour chaque préposition dans une autre langue - dans pour /v/, sur pour /na/ - mais en aucun cas nous ne pouvons garantir la substitution parfaite de la préposition par cet équivalent le plus répandu. La même situation peut être traduite par les prépositions différentes selon les langues: le syntagme выйти нА Улицу (vyjti na ulicu - litt. «sortir sur la rue ») se traduira en français par sortir dans la rue, mais ВЫЙТИ НА СЦЕНУ (vyjti na scenu - litt. "sortir sur la scène ») aura pour traduction entrer en scène (=commencer le spectacle).

4 Nous acceptons le point de vue des cognitivistes en considérant que la répartition entre les prépositions / $/ \mathrm{v}$ et $/ \mathrm{na} /$ se fait non pas en fonction d'une perception des espaces réels, mais selon une reconstruction mentale qui est fonction de multiples facteurs : culturels, sociologiques, historiques, etc. ; elle se retrouve dans les oppositions (la liste est ouverte), 
où /v/ implique le plus souvent un espace perçu comme englobant (НАХодИТЬСЯ В ЛЕСу (naxodit'sja $v$ lesu - "se trouver dans la forêt ")) et /na/ évoque généralement une surface (НАХодИТьСя НА ПлОЩАДИ (naxodit'sja na ploščadi - « se trouver sur une place »)).

Les dictionnaires donnent sur comme première traduction de na, privilège accordé à des équivalences canoniques reposant sur des relations spatiales culturellement considérées comme premières et fondamentales, indépendamment de ce qu'il en est dans les textes eux-mêmes. Dans cette optique, /na/ exprime une relation entre deux objets, dans laquelle l'un est plus haut que l'autre et en contact avec lui. C'est ce que l'on trouve dans l'énoncé typique (1).

(1) ЧАШКА СТОИТ НА СТОЛЕ

Čaška stoit na stole.

Tasse - se tient (IPF 1 ) - na - table (Prép. $\left.{ }^{2}\right)$

La tasse est posée sur la table.

Les exemples (2) et (3) ont aussi une composante spatiale, ils fournissent des réponses à des questions : « Où travaille-t-il ? Où habite-t-il ? ».

(2) ИВАН РАБОТАЕТ НА ЗАВОДЕ.

Ivan rabotaet na zavode.

Ivan - travaille (IPF) - na - usine (Prép.)

Ivan travaille à l'usine.

(3) ИВАН ЖИВёТ НА ЮГЕ.

Ivan živët na Juge.

Ivan - vit (IPF) - na - Sud (Prép.)

Ivan vit dans le Sud.

Cependant, il est peu probable que la signification de /na/ puisse être décrite ici par des propriétés spatiales comme plus haut que et en contact avec. Notons que le français n'autorise pas la préposition sur dans ces cas.

Le fonctionnement des prépositions fait largement appel aux propriétés fonctionnelles des entités ${ }^{3}$ (conçues, concrètes ou abstraites, existantes, ayant existé ou pouvant exister) et des relations qu'elles instaurent entre ces entités.

Nous poserons la question de l'unité sémantique de la préposition et de son mode de représentation. Les trois questions auxquelles nous essayerons de répondre sont les suivantes :

1. Comment analyser d'une manière systématique les différentes significations d'une préposition?

2. Comment rendre compte de ce qui unifie les divers emplois et qui permet d'avoir le sentiment qu'il y a une préposition / $/$ et une préposition / na/?

3. Comment décrire les rapports entre différents emplois des prépositions russes /v/ et /na/ ?

\section{Aspects théoriques}

10 Nous évoquerons tout d'abord quelques aspects théoriques de base soulevés dans les recherches sur les prépositions par les linguistes contemporains russes ou occidentaux. Nous ne nous arrêterons que sur quelques approches s'intéressant à l'étude de la structure sémantique et de la composante fonctionnelle des prépositions, notamment, celles des linguistes français et russes (Danielle Leeman, Claude Vandeloise, Denis Paillard, Jean-Jacques Franckel et Olga Selivërstova). 
11 Se situant dans le cadre d'une linguistique descriptive, D. Leeman considère que le signifié de la préposition associe une certaine notion (plus sophistiquée que celle que lui attribuent les dictionnaires ou une première intuition) à une certaine fonction (elle donne à interpréter d'une certaine manière le nom qui la suit, et le complément qu'elle constitue avec lui). A ce propos, D. Leeman parle d'instruction: la préposition serait un indice orientant (ou contraignant) l'interprétation ${ }^{4}$. La valeur de la préposition ne peut donc s'appréhender qu'à travers les relations paradigmatiques et syntagmatiques observables en discours. Méthodologiquement, le principe de base de D. Leeman est que l'on ne peut parvenir à une hypothèse d'identité sémantique d'une préposition qu'en procédant « au cas par cas ", faute de pouvoir saisir dans tous leurs aspects ses divers emplois à la fois.

12 La sémantique cognitive ${ }^{5}$ défendue par Claude Vandeloise s'appuie sur une analyse fonctionnelle pour caractériser les prépositions françaises, notamment, dans et sur. Il décrit la relation de localisation qui rapporte la cible (objet localisé) au site (localisateur), et les prépositions y jouent un rôle éminent.

13 Dans le cas de la préposition dans, l'accès au signifié ne peut se faire sans les notions de contenant/contenu et cela, en établissant une correspondance entre la description linguistique de l'espace et la connaissance extralinguistique que nous en avons. Mais celle-ci n'est pas objective : elle suppose une interprétation, une mise en relation des entités situées par l'institution d'une fonction de l'une par rapport à l'autre. Ainsi, le lien établi par la préposition ne décrit-il pas la réalité elle-même, mais la vision que nous en avons. Cette constatation autorise une approche onomasiologique, qui s'appuie sur une représentation de l'espace et des relations qui y existent et qui s'interroge ensuite sur les moyens linguistiques qui permettent leur expression.

14 Les linguistes Denis Paillard et Jean-Jacques Franckel considèrent la préposition comme un élément de relation, c'est-à-dire, un élément qui met en relation deux entités n'ayant pas le même statut. Selon eux, la caractérisation de la préposition comme relateur est neutre pour ce qui est des différentes valeurs : spatiales, temporelles et figurées. Les auteurs pensent qu'il est difficile de défendre la thèse de la primauté des valeurs spatiales sur les autres valeurs car il n'existe pas de critères opératoires permettant de distinguer les valeurs spatiales ou temporelles.

15 J.-J. Franckel et D. Paillard donnent comme exemple l'énoncé ambigu, où le mot Paris peut être considéré comme une ville mais aussi comme un projet : Paul travaille sur Paris. Quelle que soit l'interprétation de la séquence (zone d'activité professionnelle ou objet d'étude), sur ne dit rien de la localisation de Paul. En effet, dans Paul travaille sur Paris, ce dont Paris est localisateur (en tant que nom de ville) n'est pas Paul, mais « l'activité de Paul », d'où la différence très importante avec Il travaille à Paris.

Les auteurs proposent d'aborder les emplois fonctionnels (liés à la rection verbale) des prépositions françaises dans le cadre d'une approche unitaire des prépositions qui remet en cause la thèse de la désémantisation, ou de la décoloration des prépositions réduisant ces dernières au statut de mot-outil au service du verbe $e^{6}$. Ils partent de l'hypothèse qu'une préposition est un relateur $\mathrm{R}$ mettant en rapport deux termes $\mathrm{X}$ et $\mathrm{Y}$. Dans cette relation de repérage $e^{7}, \mathrm{Y}$ est source de détermination pour X. Si Y peut être aisément identifié au GN introduit par la préposition, $\mathrm{X}$ pose davantage de problèmes d'identification.

17 Le terme relatum ( = repère $\mathrm{Y}$ ) est utilisé également par Olga SelivËrstova. Mais dans son approche spatialisante, différente de celle de J.-J. Franckel et D. Paillard, le repère est représenté par un espace dont les composantes, fonctions, caractéristiques ou propriétés 
indiquent les coordonnées déterminant la position d'un objet-référent $\mathrm{X}$ ( = repéré $)^{8}$. Le choix de ces coordonnées varie en fonction du caractère du lien entre $\mathrm{X}$ et $\mathrm{Y}$.

O. SelivËrstova emprunte le terme imagery schemes aux cognitivistes (George Lakoff 9 ) pour expliquer la formation de certaines significations des prépositions /v/ et /na/, mais l'interprète différemment. Elle inclue dans ce terme les liens supposés entre des objets qui ne révèlent en aucun cas la nature sémantique de ces derniers ${ }^{10}$. Un tel schéma peut comprendre plusieurs significations d'un objet et être source de formation de nouvelles interprétations sémantiques en se propageant sur d'autres types d'objets. Il s'agit d'une image associative qui est à la base de plusieurs significations des prépositions reliant $\mathrm{X}$ à $\mathrm{Y}$.

Nous considérons qu'il aurait été plus judicieux de se détourner de la primauté du spatial, en assignant un rôle plus important aux éléments contextuels dans l'interprétation des prépositions. À notre avis, il serait préférable d'attribuer aux prépositions une signification unique abstraite et construire des différentes interprétations, spatiales et non spatiales, en combinant cette signification abstraite à certains éléments du contexte. Nous poserons donc une relation virtuelle :

$\mathrm{X}$ (le repéré) + Préposition $+\mathrm{Y}(\text { le repère })^{11}$.

Le premier niveau d'analyse de /v/ et /na/ sera défini par la description schématique propre à chaque préposition. Nous essayerons ensuite d'inscrire cette forme de base dans le schéma idéographique composé des concepts abstraits différenciant la sémantique d'une préposition de l'autre. Ainsi, dans notre démarche, combinant monosémie et polysémie, nous essayerons d'expliquer à partir des distributions possibles le fonctionnement des prépositions russes /v/ et /na/ en les représentant sous forme de configuration idéographique ${ }^{12}$ (CI) dans laquelle un nombre de concepts variables d'une préposition à l'autre se met en dépendance hiérarchique et s'active différemment selon le contexte discursif.

\section{Configuration idéographique (Cl)}

Chaque élément (concept) positionné en haut de cette $\mathrm{CI}$ correspondrait à un idéogramme (I) et serait lié à certains aspects (A) de son champ d'action. Ainsi, tous les éléments de la configuration auraient une signification. Les prépositions entrant dans des expressions relationnelles, en général, déclenchent l'image d'interconnexion entre les entités ( $\mathrm{X}$ et $\mathrm{Y}$ ).

Étant dynamiques, les idéogrammes ne sont pas identiques d'un emploi à l'autre de la préposition. En effet, le nombre d'aspects ou de traits activés dans le discours reste variable, ce qui explique la polysémie. C'est notre activité intellectuelle qui permettrait l'interprétation sémantique de la préposition dans le contexte, en faisant un va-et-vient permanent entre le concept et l'enchaînement singulier des faits, c'est-à-dire, entre l'espace potentiel de notre configuration idéographique (CI) et l'espace concret du syntagme réalisé dans le discours.

23 En construisant la $\mathrm{CI}$ pour chacune des prépositions étudiées à partir des distributions analysées, on dessine un plan, un modèle pouvant s'appliquer à d'autres emplois. Ce modèle reste néanmoins modifiable, perpétuellement remis en scène. La $\mathrm{CI}$ est très proche du symbole, ses concepts contiennent des images (se traduisant par des actions) qui expliquent et modélisent l'interprétation de la préposition dans le discours. 

exige d'examiner le maximum d'emplois des prépositions concernées pour extraire un principe généralisable à tous, en confrontant constamment l'hypothèse de départ à un autre emploi. L'abstraction que suppose la FSB consiste à extraire un type d'interaction entre le repéré $X$ et le repère $Y$ (ou entre ses deux strates $Y 1$ et $Y 2$ ). Ainsi, nous allons appeler la FSB de /v/ La forme schématique d'inclusion:

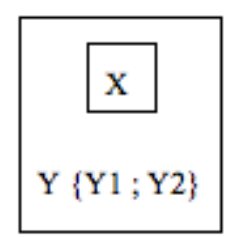

Quant à la FSB de /na/, elle sera nommée La forme schématique de superposition :

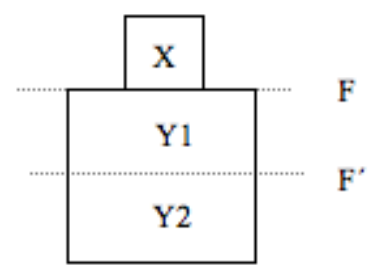

Dans ces FSB des prépositions russes /v/ et /na/, nous distinguons deux entités X (le repéré), $\mathrm{Y}$ (le repère). Ainsi, $\mathrm{X}$, une entité (conçue, imaginée, concrète ou abstraite) est localisée dans le temps et l'espace par rapport à une autre entité $Y$ dont les composantes, fonctions, caractéristiques ou propriétés symbolisent les coordonnées déterminant la position de X.

30 La frontière $\mathrm{F}$, présente uniquement dans la FSB de /na/, sépare les domaines notionnels ${ }^{13}$ de $\mathrm{X}$ et $\mathrm{Y} 1$, alors que $\mathrm{F}^{\prime}$ dissocie le domaine $\mathrm{Y} 1$ de celui de $\mathrm{Y} 2$. 
31 Ainsi, nous supposons l'existence d'une seconde frontière $F^{\prime}$ dans la FSB de /na/. Cette frontière $F^{\prime}$ découpe le domaine $\mathrm{Y}$ en deux parties $\mathrm{Y} 1$ et $\mathrm{Y} 2$. Donc, $\mathrm{Y}$ a une double structure sémantique. Et c'est la superposition, le trait relationnel principal de la préposition /na/, qui traduit ce dédoublement de Y marquant l'ajout de l'espace temporel (événementiel) (Y2) à l'espace géométrique (topologique) (Y1) et / ou décrivant la position de $\mathrm{X}$ « par-dessus » $\mathrm{Y}\{\mathrm{Y} 1 ; \mathrm{Y} 2\}$. Ainsi, nous considérons qu'il y a dans le cas de / na/ deux hypostases de $Y: Y 1$ et $Y 2$.

L'exemple ci-dessous montre la différence dans la perception de la frontière entre Y1 et Y2 en russe et en français. Là où le français utilisera la préposition dans (ou à), le russe préférera la préposition /na/ :

(4) ЮЛЯ НА ВОСЬМОМ МЕСЯЦЕ БЕРЕМЕННОСТИ.

Julja na vos'mom mesjace beremennosti.

Julja - na - huitième (Prép.) - mois (Prép.) - grossesse (Gén.)

Julie est dans (Julie en est à) son huitième mois de grossesse.

Cet énoncé décrit un état en cours (Y1) par rapport à la visée (Y2). Ces deux stades se définissent respectivement comme le stade de grossesse, qui est ici exprimé par son huitième mois de grossesse (Y1) dans lequel se trouve X, et le stade de l'accouchement (Y2), qui est implicite dans l'énoncé, extérieur à la position de $\mathrm{X}$. Le locuteur précise à quelle étape de sa grossesse se trouve Julie, sachant qu'elle est dans un état visant l'accouchement. L'énoncé russe part de la présupposition que X (Julie) se trouve dans une période possédant une limite, limite qui n'est conçue que compte tenu de la visée. Autrement dit, l'énonciation est basée sur la frontière $\left(\mathrm{F}^{\prime}\right)$ entre deux stades $\mathrm{Y} 1$ et $\mathrm{Y} 2$.

$\mathrm{Si}$ en français, la préposition dans marque la relation d'attachement de $\mathrm{X}$ à la période limitée $\mathrm{Y}$, en russe, /na/ tient compte davantage du passage potentiel de X à Y2 via Y1. Ainsi, юля(Julie) parcourt les mois de grossesse dans un seul but: arriver à l'état d'accouchement, c'est-à-dire, atteindre le domaine Y2. Les mois se superposent dans le temps (ou se succèdent) et Julie se retrouve chaque fois à la frontière marquant ces étapes de passage sur le chemin jusqu'à Y2.

Tout en visant $\mathrm{Y} 2, \mathrm{X}$ n'est pas enfermé dans un domaine limité comme dans l'exemple en français, mais se place à la frontière entre le huitième et le neuvième mois de grossesse considérés comme points de passage, éléments parmi d'autres dans une suite spatiotemporelle. Ceci est comparable au cas d'arrêts de bus, où le russe et le français emploient les prépositions /na/ et à (ОЖИДАТЬ КОГО-ЛИБО НА АВТОБУСНОЙ ОСТАНОВКЕ (оžidat' kogo-libo na avtobusnoj ostanovke - "attendre qqn à l'arrêt de bus»)), et concerne le grand nombre de compléments dits «de temps» associant/na/ à un ordinal (нА ПЕРВОЙ МИНУТЕ Боя (na pervoj minute boja - "à la première minute du combat »)) ou un terme équivalent (послЕдний (poslednij - « dernier »), par exemple).

\section{Construction de la configuration idéographique de la préposition /na/}

\subsection{Distinction : repère-support / repère-but}

En reprenant notre schéma de base (FSB) pour la préposition /na/, notons qu'il s'inscrit dans la situation du mouvement spatio-temporel qui a plusieurs variantes significatives. 
Chacune de ces variantes donne ses propres « réflexes ${ }^{14}$ sémantiques (c'est-à-dire, produit des syntagmes au contenu sémantique différent).

Nous choisissons d'examiner la relation X /NA/ Y + Acc. ${ }^{15}$ en mettant en exergue d'une part :

1) La relation de prédétermination entre $X$ et $Y$-but : la présence d'une distance entre le mobile $^{16}$ et le repère, le déplacement du mobile dans la direction du repère. Grâce à l'apport de l'Accusatif directionnel, le repère est considéré dans ce cas comme un point final, un but.

Dans cette relation de but, nous devrions distinguer deux types de repères : point d'arrivée (limite) et situation future (situation visée).

A. Le repère représente le point final du déplacement, le terminus qui se réfère à un lieu ouvert que le mobile animé a pour but d'atteindre. Au point de départ, le mobile (X) ne voit pas toujours son point d'arrivée $(\mathrm{Y})$, mais sait qu'il existe et veut l'atteindre: $\mathrm{X}$ ПОШёЛ НА СТАДИОН / НА РЕЧКУ / НА ПЛЯЖ (Х роšёl na stadion (Acc.) / na rečku (Acc.) / na pljaž (Acc.) - "X est allé au stade / à la rivière / à la plage »).

B. Le repère se réfère à la situation dans le futur (limite spatio-temporelle) décrite souvent par les noms-événements: X ПРИШёЛ НА РАБОТУ / НА КОНЦЕРТ / НА СВАДЬБУ / HA СОБРАНИЕ / НА ВОЙНУ. (Х prišël $\boldsymbol{n} \boldsymbol{a}$ rabotu (Acc.) / na koncert (Acc.) / na svad'bu (Acc.) / na sobranie (Acc.) / na vojnu (Acc.) - "X est arrivé au travail / au concert / au mariage / à la réunion / à la guerre "1 ${ }^{17}$.

Dans ces énoncés, Y renvoie à un événement qui se produit dans un lieu. Il s'agit d'un repère spatio-temporel. Pour participer à cet événement, atteindre la limite posée, le mobile $\mathrm{X}$ doit effectuer un déplacement, parcourir une distance pour arriver dans le lieu Y1 où se produira l'événement Y2. La visée implique une prédétermination, trait idéographique essentiel de la préposition /na/ qui est à l'origine de la décision de $\mathrm{X}$ d'entrer en relation avec le repère $Y$. En effet, les noms-événements cités ci-dessus incluent des « actions " prédéterminées, que le mobile $\mathrm{X}$ a prévu d'effectuer dans un lieu approprié : s'il est allé au travail, c'est pour y travailler, s'il est venu à un concert c'est pour écouter des artistes, s'il s'est déplacé à un mariage, c'est pour participer ou assister à la fête ${ }^{18}$.

D'autre part, nous mettrons l'accent sur :

2) La relation de pression entre $X$ et $Y$-support : la position du mobile « au-dessus », « en haut » (= superposition), «à la surface » ( = contact) renvoyant par métaphorisation à l'idée de " pression », de " pouvoir », d'« influence » et l'emplacement du repère « au-dessous », " en bas ", évoquant l'idée de " support ».

Nous distinguons ces deux relations dans le but de spécifier la superposition de $\mathrm{X}$ par rapport à $\mathrm{Y}$ dédoublé en $\mathrm{Y} 1$ et $\mathrm{Y} 2$ dans la relation $\mathrm{X} / \mathrm{na} / \mathrm{Y}$ et le rôle de support de $\mathrm{Y}$ n'apparaissant que dans la relation de pression.

41 Au titre d'exemple, dans l'inscription sur les fusées de l'Armée Rouge « НА БЕРЛИН ! (" Na Berlin!» (Acc.)) illustrant la relation de pression, la fusée est bien destinée non seulement à aller à Berlin, mais aussi à exercer une action sur Berlin. L'idée d'action visant la possession correspond à celle de pression. Cependant, avec la préposition /v/, on perd cette image de pression: «В БЕРлин!» («V Berlin!» (Acc.)) ne marque que la direction de déplacement dans les limites d'un espace fermé (une ville) : - Вы кудА ЕдЕ ТЕ ? - В БЕРлИн ! (Vy kuda edete ? - V Berlin ! - « Où allez-vous ? - À Berlin!»). 

Y qui devient le moyen ou intermédiaire de réalisation d'une action (ех. НАДЕЯТЬСЯ НА (nadejat'sja na + Acc. - "porter l'espoir sur»), РАССЧИТЫВАТЬ НА (rassčityvat' na + Acc. " compter sur »), оснОВЫВАТЬ нА (osnovyvat' na + Acc. - « fonder sur »)). Nous y introduisons un autre trait relationnel dérivé du support - médiation qui vise à définir l'intervention d'un tiers (Y) pour faciliter ou conditionner l'action de $\mathrm{X}$.

Dans le cas de НАДЕЯТЬСЯ НА КОГО-ЛИБО (nadejat'sja na kogo-libo (Acc.) - « compter sur quelqu'un »), le verbe НАДЕЯТьСя (nadejat'sja - « espérer ») provient de *nadĕti (ПоложИТь, ПОСТАВИТЬ (položit' $\left(\mathrm{PF}^{21}\right)$ - «mettre en position horizontale», postavit' (PF) - «mettre en position verticale $») \leftarrow{ }^{*}$ dĕti (КЛАСТЬ, СТАВИТЬ (klast' (IPF), stavit' (IPF)), qui veut dire en vieux-slave mettre, poser. Le verbe НАДЕЯТЬСЯ (nadejat'sja) peut être paraphrasé par ВОЗЛАГАТЬ НАДЕЖДУ (litt. poser l'espoir). Donc, l'ancien préverbé (aujourd'hui, НАДЕ-Я-ТЬСЯ (nade-ja-t'-sja) considéré comme non préverbé) a pour rôle d'introduire $\mathrm{Y}$ - un espoir d'une action. Quant au syntagme prépositionnel НАДЕЯТЬСЯ НА КОГО-ЛИБо (nadejat'sja na kogo-libo), il peut être compris comme "se poser / compter sur quelqu'un », où la préposition /na/ introduit en plus l'auteur (Y1) (quelqu'un) d'une action (Y2) attendue par celui qui espère sa réalisation $(\mathrm{X})$ (le sujet de l'énoncé).

Prenons trois autres exemples : 
(5) Я ВЕРЮ ЕМУ.

Ja verju emu.

Je - crois (IPF) - il (Dat.)

Je le crois.

(6) Я ВЕРЮ В НЕГО.

Ja verju $\mathbf{v}$ nego.

Je - crois (IPF) - v - il (Acc.)

Je crois en lui.

(7) Я РАССЧИТЫВАЮ НА НЕГО.

Ja rassčityvaju na nego.

Je - compte (IPF) - na - il (Acc.)

Je compte sur lui.

Le premier exemple montre que X (je) n'a aucun doute en ce qui a été dit par Y (il). Le deuxième exemple affirme que $\mathrm{X}$ croit qu'Y possède des qualités lui permettant de réussir dans tout ce qu'il entreprend. La préposition /v/ limite le champ d'existence de ces qualités en ce qu'elles ne doivent être propres qu'à $Y$ lui-même. Enfin, le troisième exemple marque aussi la confiance de $\mathrm{X}$ en $\mathrm{Y}$, mais $\mathrm{Y}$ est présenté en plus comme celui qui doit aider ensuite $X$ dans la réalisation de quelque chose. C'est le support ou l'outil dont $X$ a besoin pour effectuer une action.

\subsection{Prédétermination ou spécificité de /na/ par rapport à /v/}

La chose importante à noter est le fait que /na/ ne s'emploie qu'avec les prédicats qui incluent $\mathrm{Y}$ dans le programme de futures actions de X (ех. СоглАситься нА (soglasit'sja na + Acc. - "être d'accord pour qqch»), mais ОТКАЗАТЬСЯ от (otkazat'sja ot + Gén. - "refuser $q q c h »)$ ). Remarquons que $X$ peut jouer le rôle de sujet dans la phrase (ex. X РЕшился НA $Y$ (X rešilsja na $Y(A c c)$. - «X s'est décidé à effectuer $Y$ ») ou celui de COD (Z ПодзАдОРИЛ X НА $Y$ (Z podzadoril X (Acc.) na $Y($ Acc.) - « Z a poussé X à effectuer $Y »)$.

La préposition /na/ établit le rapport entre le mobile $X$ et l'événement futur $Y$ tel qu'on le "prévoit ", qu'on l'a planifié, attendu, désiré. Ainsi, le repère Y correspond à une situation dont le temps et le lieu sont déterminés par avance. C'est-à-dire, la préposition / $\mathrm{na} /$ sert à décrire une relation prédéterminée entre $\mathrm{X}$ et $\mathrm{Y}$. Cette particularité du schéma idéographique de /na/ se manifeste dans tous les contextes : ЗАПИСАться нА ПРИём К ВРАЧУ (zapisat'sja na priëm $k$ vraču (s'inscrire - na - rendez-vous (Acc.) - chez - medecin (Dat.)) "prendre rendez-vous chez le médecin»); КУПИТЬ ШЕРсть нА Костюм (kupit' šerst' na kostjum (acheter - laine (Acc.) - na - tailleur (Acc.)) - «acheter un tissu de laine pour le tailleur »).

Il est important de noter que la situation Y n'est pas prédéterminée par le mobile luimême, mais par l'observateur non participant, extérieur à la relation X/NA/ Y. Si la réalisation de l'événement $\mathrm{Y}$ dépend de $\mathrm{X}$ et peut être modifiée par lui, la préposition / na/ n'est pas employée. Ainsi, l'énoncé :

(8) ИВАНОВ ПОШёЛ НА ОБЕД.

Ivanov pošël na obed.

Ivanov - est allé - na - déjeuner (Acc.)

Ivanov est allé à un déjeuner.

devrait être interprété comme :

(8a) [...] НА ОБЕДЕННЫЙ ПЕРЕРЫВ.

[...] na obedennyj pereryv.

[...] na - de déjeuner (Adjectif) - pause (Acc.)

Ivanov a pris sa pause-déjeuner. 
(8b) ИВАНОВа ПРИГЛАСИЛИ НА ОБЕД

Ivanova priglasili na obed.

Ivanov (Acc.) - ont invité - na - déjeuner (Acc.)

Ivanov a été invité à un déjeuner.

Il est incorrect de dire Пошёл НА ОБЕД (pošël na obed (Acc.) - «il est allé à un déjeuner ») en parlant du déjeuner (Y) préparé par ses propres soins. Si l'on évoque «son propre déjeuner », « le déjeuner chez soi », on emploie la construction infinitive пошёл ОБЕдАТЬ (pošël obedat' - «il est allé déjeuner »).

53 Le trait sémantique qui permet d'expliquer le choix entre la construction infinitive ПОШЕЛ ОБЕДАТЬ (il est allé déjeuner) et la construction prépositionnelle ПОШёЛ НА ОБЕД (il est alléà un déjeuner) est l'existence ou l'absence d'un contrôle exercé par le sujet (X) sur sa relation à l'entité moins saillante, plus abstraite - déjeuner (Y). Dans la construction infinitive, l'action déjeuner est traitée comme élément de la sphère personnelle du sujet, ce dernier étant à la fois le participant et le contrôleur de l'action. Dans la structure prépositionnelle, l'entité déjeuner $(\mathrm{Y})$ introduite par la préposition /na/ n'est plus entièrement contrôlée par le sujet $(\mathrm{X})$, donc, ne relève plus de sa sphère personnelle.

54 À la différence de /na/, qui n'implique pas la participation de $\mathrm{X}$ dans l'événement $\mathrm{Y}$, la préposition / $\mathrm{v} /$ oblige $\mathrm{X}$ à prendre part dans l'événement $\mathrm{Y}$ en s'introduisant au fond de son domaine. C'est pour cette raison que l'on ne peut pas dire en russe *HA АTAKУ! $\left({ }^{*} \mathrm{Na}\right.$ ataku (Acc.)!), mais B ATAKY ! (V ataku (Acc.)! - « À l'attaque!»). En effet, l'attaque fait déjà partie du combat. Donc, $\mathrm{X}$ est forcément à l'intérieur de $\mathrm{Y}$.

5 La préposition /na/marque seulement la présence, l'existence de $\mathrm{X}$ mais non pas sa participation active à l'événement $\mathrm{Y}$. Cela n'exclut pas la participation éventuelle de $\mathrm{X}$ dans l'événement Y. Ainsi, l'énoncé АРТИСТЫ ПРИШЛИ НА СПЕКТАКЛЬ (artisty prišli na spektakl' (Acc.) - «les artistes sont venus au spectacle ») peut être périphrasé : les artistes sont présents au spectacle et il est possible qu'ils vont y jouer. Cependant, ce n'est pas la préposition /na/ mais d'autres éléments du contexte qui marquent cette participation de X à Y. La préposition /na/ n'est responsable que d'une simple présence de $X$ «à la surface» du domaine $Y$, elle n'embrasse pas la partie intérieure, profonde de $Y$, où les choses entrent en action.

C'est probablement pour cette raison que /na/ s'emploie fréquemment avec les nomsévénements organisés, préparés, et $/ \mathrm{v} /$ décrit mieux les relations dans les situations naturelles, totalement ou partiellement incontrôlables : Вступить в Борььу / в игру / в ПЕРеБРАНКУ (vstupit' v bor'bu (Acc.) / v igru (Acc.) / v perebranku (Acc.) - «entrer en guerre / en jeu / dans la dispute»).

57 Une caractéristique importante de la préposition /na/ est sa capacité d'introduire des éléments nouveaux dans la structure sémantique de l'énoncé (cf. Быть на испытАниях (byt' na ispytanijax - «assister aux épreuves ») vs. Быть в испытаниях (byt' v ispytanijax «subir les épreuves»)). Ainsi, /na/, en plus de son rôle de relateur qui relie deux entités entre elles prend des valeurs sémantiques supplémentaires : par exemple, l'indication de lieu aux noms-événements (cf. ispytanija) en créant un espace composé, dénoté par Y1 (lieu des épreuves dans lequel se trouve $\mathrm{X}$ ) et Y2 (moment de réalisation des épreuves auxquelles assiste (mais ne participe pas) $\mathrm{X}$. 


\subsection{Bilan : configuration idéographique de la préposition /na/}

\section{Après avoir analysé plusieurs exemples, on aboutit à une configuration composée des} traits idéographiques hiérarchisés et interdépendants de la préposition /na/ :

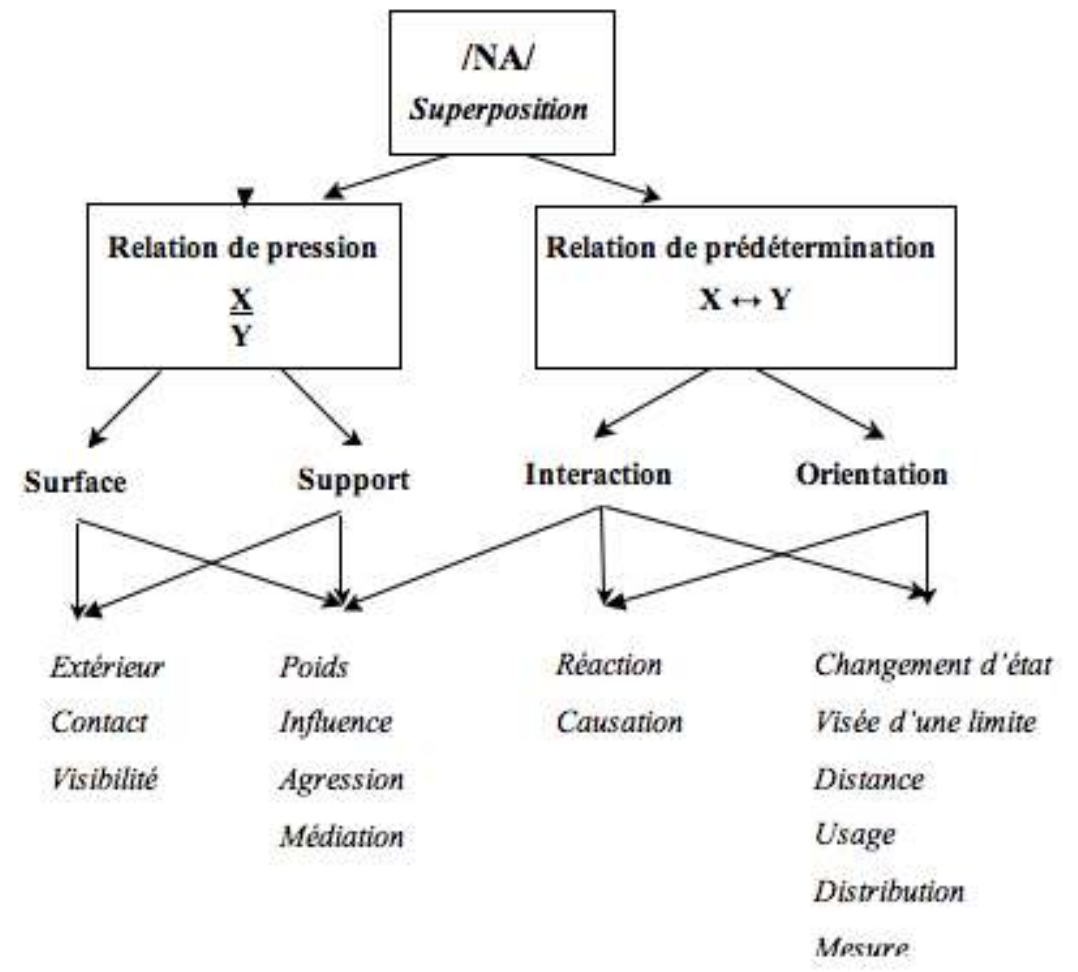

Classification d'après le trait dominant :

1) SURFACE :

+ Activation du trait extérieur:

HА ДВОРЕ МОРОЗ - il gèle dehors

na dvore moroz = na - cour (Prép.) - gelée (Nom.)

+ Activation du trait contact:

ЧАСЫ НА РУКе - lA montre au poignet

časy na ruke $=$ montre $($ Nom. $)-$ na - main (Prép. $)$

положить нА стол - poser sur la table

položit' na stol $=$ mettre (en position horizontale) $(\mathrm{PF})-\mathbf{n a}-$ table (Acc.)

+ Activation du trait visibilité (exposition):

ЛЕЖАТЬ НА СОЛНЦЕ - être allongé au soleil

ležat' na solnce = être allongé (IPF) - na - soleil (Prép.)

2) SUPPORT :

+ Activation du trait poids :

ВЗВАЛИТЬ МЕШОК СЕБЕ НА СПИНУ - se mettre un sac sur le dos vzvalit' mešok sebe na spinu = charger (PF) - sac - soi-même (Dat.) - na - dos (Acc.)

+ Activation du trait influence:

ДЕЙСТВОВАТЬ НА КОГО-ТО - agir sur quelqu'un

dejstvovat' na kogo-to = agir (IPF) - na - quelqu'un (Acc.)

+ Activation du trait agression: 
КРИЧАТЬ НА РЕБЁНКА - crier sur un enfant

kričat' na rebënka = crier (IPF) - na - enfant (Acc.)

+ Activation du trait médiation:

ЕХАТЬ НА ПАРОХОДЕ - aller en bateau

exat' na paroxode = aller (IPF) - na - bateau à vapeur (Prép.)

$613)$ INTERACTION :

+ Activation du trait réaction:

ОТВЕТИТЬ НА ВОПРОС - répondre à une question

otvetit' na vopros = répondre $(\mathrm{PF})$ - na - question (Acc.)

+ Activation du trait causation:

ОБИДЕТЬСЯ НА ДРУГА - s'offenser contre un ami

obidet'sja na druga= s'offenser (PF) - na - ami (Acc.)

4) ORIENTATION :

+ Activation du trait changement d'état :

ПЕРЕВЕСТИ НА РУССКИЙ ЯЗЫК - traduire en russe

perevesti na russkij jazyk = traduire $(\mathrm{PF})-\mathbf{n a}-$ russe (Adjectif Acc.) - langue (Acc.)

+ Activation du trait visée d'une limite :

СПЕШИТЬ НА ПОЕЗД - se dépêcher pour prendre le train

spešit' na poezd = se dépêcher (IPF) - na - train (Acc.)

+ Activation du trait distance :

ПОЕЗД НА МОСКВУ - le train pour Moscou

poezd na Moskvu = train - na - Moscou (Acc.)

+ Activation du trait usage:

САПОГИ НА ЗИМУ - les bottes pour l'hiver

sapogi na zimu = bottes - na - hiver (Acc.)

+ Activation du trait distribution:

УЖИН НА ПЯТЬ ЧЕЛОВЕК - dîner pour cinq personnes

užin na pjat' čelovek = dîner $($ Nom. $)-$ na - cinq (Acc.) - personnes (Gén.)

+ Activation du trait mesure :

ПРИЕХАТЬ НА ДВА ДНЯ - venir pour deux jours

priexat' na dva dnja = venir (PF) - na - deux (Acc.) - jour (Gén.)

ОТСТУПИТЬ НА ДВА ШАГА - reculer de deux pas

otstupit' na dva šaga = reculer $(\mathrm{PF})-$ na $-\operatorname{deux}($ Acc.) - pas (Gén. sg.)

\section{Configuration idéographique de la préposition /v/}

\subsection{Contenance et idéalisation du repère}

63 La seconde préposition à laquelle nous nous intéressons dans notre article, la préposition /v/ $\left(/ \mathrm{vo} /{ }^{22}\right)$, sera étudiée essentiellement par opposition à /na/. Nous essayerons d'expliquer notamment l'emploi de la préposition /v/ dans la relation d'emplacement et de déplacement de X par rapport à Y. Si dans l'analyse de /na/ nous avons mis l'accent sur le rôle du repère $Y$ (support/but) dans la relation de pression ou de prédétermination et sa double structure $(\mathrm{Y} 1+\mathrm{Y} 2)$ déterminant la superposition des domaines notionnels de $\mathrm{X}$ et $Y$, la description de /V/ sera centrée sur le processus même (ou son état résultatif) mettant en rapport $\mathrm{X}$ et $\mathrm{Y}$. 

Y. Du point de vue fonctionnel, cette inclusion peut être partielle (ключ в ЗАмке́ (ključ v zamke (Prép.) - « la clé dans la serrure »), ГВОЗДЬ В СТЕНЕ (gvozd’ v stene (Prép.) - «le clou dans le mur »), mais concerne les repères qui acceptent une introduction quelconque dans une partie de son espace intérieur. C'est pourquoi le russe n'admet pas *КАСтРюЛЯ в КРЫшКЕ (*kastrjulja v kryške (Prép.) - " *la casserole dans le couvercle), *ПОСЫЛКА В ВЕРёВКЕ (posylka v verëvke - "*le colis dans la corde ») où les repères кРЫшка et ВЕРёвка fonctionnent comme des objets non contenants, n'ayant pas de cavité à remplir.

Cependant, nous nous accordons avec Claude Vandeloise sur le point que les critères topologiques n'expliquent pas toujours la relation d'inclusion, et qu'il faut par conséquent en venir à des critères fonctionnels, en s'intéressant au potentiel des repères pouvant sous certaines conditions marquer une relation d'inclusion dans un domaine tridimensionnel, volumineux (ПтИЦА В КЛЕТКЕ (ptica v kletke (Prép.) - «l'oiseau dans la cage »), ПОЕЗД В ТУННЕЛЕ (poezd v tunnele (Prép.) - «le train dans le tunnel »)).

Notons que le critère géométrique de tridimensionnalité évoqué pour l'emploi de /v/ n'est qu'une conséquence de la signification de cette préposition qui repère un terme comme contenu dans un autre, le contenant. La condition pesant sur le second étant d'être un contenant potentiel, on peut s'attendre à ce que les objets à trois dimensions ou même à deux satisfassent plus souvent ou plus typiquement à cette condition que les objets à une dimension.

préposition /v/ est relativement indifférente à la dimensionnalité de son complément. En français, d'ailleurs, on n'a même pas besoin de recourir à une préposition pour décrire ce contexte: ils font la queue. Par conséquent, on peut parler du phénomène très général d'idéalisation du repère saisi par une préposition. Ainsi, tout objet verbal peut, à la limite, être conçu comme adimensionnel.

Il est important de noter que les significations abstraites obtenues lors des transferts métaphoriques des prépositions / $/ \mathrm{l}$ et /na/ conservent leurs traits de base, à savoir, le trait d'inclusion pour /v/ et le trait de support pour /na/. En effet, НАПИСАТЬ В ГАЗЕТЕ (napisat' v gazete - "écrire dans le journal») c'est placer à l'intérieur ou inclure une information dans le journal, ПРИЙТИ В КУРТКЕ (prijti v kurtke - "venir avec une veste») renvoie en russe au corps humain se trouvant «à l'intérieur » de la veste, гордость В сыне (gordost' $v$ syne - «la fierté dans le fils») implique le sentiment de fierté inclus dans l'ensemble des qualités propres à la personne, ici le fils. De même, avec la préposition / na/ nous avons ЕХАТЬ НА АВТОБУСЕ (exat' na avtobuse - «aller en bus») qui renvoie à un «objet-support » dans le but de spécifier le mode de déplacement; РАЗГОВАРИВАТЬ НА КУХНЕ (razgovarivat' na kuxne - "discuter dans la cuisine ») qui demande de considérer la cuisine non pas comme une simple pièce de l'appartement mais comme un endroit fonctionnel, un support pour les "objets " qui rendent à cette pièce son utilité (la cuisinière, la table à manger, le vaisselier, etc.).

\subsection{Relation directionnelle $: v+$ Accusatif}

69 Les deux champs fonctionnels dans lesquels s'inscrit la préposition /v/ - la position et la direction - se traduisent par le marquage casuel (le Prépositif et l'Accusatif) et se trouvent interdépendants en partageant et échangeant leurs traits relationnels. Si la préposition /

Corela, 9-1 | 2011 
na/ marquait en termes de déplacement l'orientation vers la surface d'un objet, la préposition /v/ indique la direction précise vers l'intérieur de l'objet. Le résultat du déplacement du mobile dans la direction du repère est l'emplacement de $\mathrm{X}$ dans les limites du domaine Y. Ainsi, le Prépositif a pour fonction de mettre l'accent sur le résultat de l'action: il montre que le repéré trouve sa place dans le repère. L'Accusatif, quant à lui, met l'accent sur le processus même et la direction que doit suivre le repéré pour arriver au repère.

70 Il est nécessaire de distinguer les fonctions des prépositions et celles des cas puisque d'une part, de différentes prépositions peuvent régir le même cas, et d'autre part, la même préposition peut être suivie des formes casuelles différentes. À travers des propriétés casuelles différenciées, on peut expliquer au moins partiellement les différents comportements grammaticaux de variantes prépositionnelles en russe. Nous nous intéressons au marquage casuel, au régime des prépositions étudiées $/ \mathrm{v} /$ et $/ \mathrm{na} /$, afin de voir comment les cas modifient le nombre de traits activés en influençant ainsi notre interprétation de la relation prépositionnelle entre $\mathrm{X}$ et $\mathrm{Y}$.

71 Suivie de l'Accusatif, /v/ exprime la direction ou l'arrivée d'un(e) objet (personne) à un point final précis. Ainsi, la construction prépositionnelle correspond à la structure en direction de) : ДОРОГА В ГОРОД (doroga $v$ gorod (Acc.) - «la route pour la ville (menant à la ville) »), ОКНА ВО ДВОР (okna vo dvor (Acc.) - «les fenêtres donnant sur la cour »), НОСОМ В угол (nosom v ugol (Acc.) - «le nez dans le coin »).

La notion de direction précise est à distinguer de la visée marquée par la préposition /na/. En effet, doroga $v$ gorod a un point d'aboutissement précis - une ville, et suppose l'entrée $\mathrm{du}$ sujet qui suit cette route dans un domaine notionnel $Y$ : espace indivisible de la ville coïncidant avec le point de destination finale du sujet.

Quant à doroga na gorod, elle oriente le sujet sur le trajet qui mène à la ville, cette ville dans laquelle le sujet n'arrivera pas obligatoirement (il pourrait s'arrêter dans un village avant cette ville, par exemple) : son point d'arrivée (Y2) ne coïncide pas avec l'espace de la ville (Y1). Ainsi, la préposition /na/ se réfère à une étape quelconque d'un chemin «spatial » ou « temporel », alors que la préposition /v/ marque plutôt ses points extrêmes, le début ou la limite finale.

74 Cela explique le besoin de déterminer le repère gorod lorsqu'il s'emploie avec /na/ (gorod est alors souvent accompagné du nom de la ville, ou s'oppose à l'existence d'une autre direction). Il devient intéressant de remplacer gorod par Berlin, et de comparer doroga $v$ Berlin / doroga na Berlin :

(9) ТАМ ОСТАЁТСЯ ЕЩЁ СТОЛЬКО НЕОБСЛЕДОВАННЫХ УГОЛКОВ И НЕВЕРОЯТНЫХ КРАСОТ, НО НАС ЖДЁТ УЖЕ ДОРОГА В БЕРЛИН. (S. Ben' “Serebrjanaja svad'ba », 2009)

Tam ostaËtsja ešč ždËt uže doroga v Berin.

Là-bas - il reste (IPF) - encore - inexplorés (Gén.) - coins (Gén.) - et - incroyables (Gén.) - beautés (Gén.) - mais - nous (Acc.) - attend (IPF) - route (Nom.) - v - Berlin (Acc.)

Il nous reste encore tellement de lieux et de beautés insolites à explorer, mais la route pour Berlin nous attend déjà.

(10) И ВСЕ-ТАКИ У ЛЮДЕЙ ЖИЛА НАДЕЖДА, ЧТО ВДРУГ ЗАВТРА МОЖЕТ СРАЗУ ПРИЙТИ ПОЛНАЯ ПОБЕДА, И ДОЛГАЯ ДОРОГА НА БЕРЛИН ПРЕВРАТИТСЯ В КОРОТКУЮ. (Rejnov N., Volodin B. «Ximija $i$ žizn'" , 1970)

I vsË-taki u ljudej žila nadežda, čto vdrug zavtra možet srazu prijti polnaja pobeda, $\mathrm{i}$ dolgaja doroga na Berlin prevratitsja v korotkuju. 
Et - tout de même - chez - gens (Gén.) - vivait (IPF) - espoir (Nom.) - que - soudain - demain - peut (IPF) - arriver (PF) - tout d'un coup - complète (Nom.) - victoire (Nom.) - et - longue (Nom.) - route (Nom.) - na - Berlin (Acc.) - se transforme (PF) $-\mathbf{v}$ - courte (Acc.).

Tout de même, les gens gardaient l'espoir qu'une victoire complète pourrait arriver tout d'un coup, et qu'un long chemin pour (à la conquête de) Berlin se raccourcirait.

Dans l'énoncé (9), doroga $v$ Berlin est déterminée précisément par son point de destination : la route $(\mathrm{X})$ conduit et aboutit à Berlin (Y). Le point d'arrivée du locuteur (nous) coïncide avec le point final de la route : le locuteur veut aller à Berlin et il prend précisément la route qui y mène. L'exemple (10) est différent : doroga $\boldsymbol{n} \boldsymbol{a}$ Berlin est prise dans son étendue ou sa durée (d'où l'ajout des adjectifs qualificatifs dolgaja, korotkuju). Par conséquent, nous avons préféré de traduire doroga par chemin renvoyant mieux à cette idée de parcours en vue d'une conquête de la ville, d'une victoire espérée sur ses habitants. Ainsi avec /na/, ce n'est pas le point d'arrivée à Berlin (Y1) qui prime, mais le parcours même (Y2) lié métaphoriquement à la guerre avec l'Allemagne.

\subsubsection{Rapidité et coïncidence}

Le trait coïncidence se rapporte aux événements. Dans le contexte temporel, nous relevons les expressions qui utilisent les prépositions composées (Во ВРЕмя (vo vremja (Acc.) «pendant »), В ПРОДОЛЖЕНИЕ (v prodolženie (Acc.) - « tout le long de »), В ТЕЧЕНИЕ (v tečenie (Acc.) - « au cours de ») pour marquer un événement coïncidant dans le temps avec l'action exprimée par le verbe :

(11) НА ТЕРАССЕ В ТЕЧЕНИЕ НЕСКОЛЬКИХ МГНОВЕНИЙ ГОСПОДСТВОВАЛО МОЛЧАНИЕ.

Na terasse $\mathbf{v}$ tečenie neskol'kix mgnovenij gospodstvovalo molčanie.

$\mathrm{Na}$ - terrasse (Prép.) - au cours de - quelques (Gén.) - instants (Gén.) - régnait -

silence.

Pendant quelques petits instants un silence régna sur la terrasse.

77 La coïncidence pourrait être traduite dans cet exemple de façon suivante : la durée du silence qui régnait correspond à la durée de quelques petits instants passés sur la terrasse.

Une nuance supplémentaire de rapidité de l'action (souvent au sens de plus vite que l'on ne croyait) s'ajoute à la coïncidence dans les constructions avec /v/ qui diffèrent de celles avec $/ \mathrm{za} /{ }^{23}$ :

(12a) ОН РЕШИЛ ЗАДАЧУ В ДВЕ МИНУТЫ.

On rešil zadaču $\mathbf{v}$ dve minuty.

Il - a résolu (PF) - problème (Acc.) - v - deux (Acc.) - minutes (Gén. sg.)

Il a résolu le problème en deux minutes.

(12b) ОН РЕШИЛ ЗАДАЧУ ЗА ДВЕ МИНУТЫ.

On rešil zadaču za dve minuty.

Il - a résolu (PF) - problème (Acc.) - za - deux (Acc.) - minutes (Gén. sg.)

Il a résolu le problème en deux minutes.

79 Ces constructions sont traduites en français par les expressions avec la préposition en n'exprimant qu'une simultanéité absolue (ou coïncidence) sans aucune nuance supplémentaire. Par contre, en russe, le choix de la préposition /v/ oppose le temps normal (tel qu'on l'imagine) nécessaire à la réalisation d'une action (Y2) au temps effectif, beaucoup plus court, pendant lequel l'action a réellement eu lieu (Y1). 
80 Ainsi, la préposition /v/ suivie de l'Accusatif souligne la vitesse, la rapidité dans le déroulement d'une action, le numéral cardinal oppose la durée habituelle (Y2) à la durée réelle (Y1) de cette action et l'aspect perfectif du verbe marque son accomplissement.

\subsection{Relation positionnelle : $v+$ Prépositif}

Nous appelons relation positionnelle emplacement de l'entité $\mathrm{X}$ à l'intérieur du domaine $\mathrm{Y}$. Cette position résulte de la relation directionnelle et se décrit au moyen de la préposition /v/ suivie du cas Prépositif (ПРЕДЛОЖНЫЙ ПАДЕЖ - «Predložnyj padež»). a préposition /na/ comptait dans sa configuration le trait liberté dû à l'absence de limites, avec /v/ dans les expressions РАссЫПАТЬСя в ПОХВАЛАХ (rassypat'sja $\boldsymbol{v}$ poxvalax (Prép.) (litt. se répandre dans les éloges) - "prodiguer des louanges»), РАссЫПАТься В ИзВИНЕНИЯХ (rassypat'sja v izvinenijax (Prép.) - «se confondre en excuses»), ТЕРЯТЬСЯ В ДОГАДКАХ (terjat'sja $\boldsymbol{v}$ dogadkax (Prép.) - «se perdre en conjectures »), ПУТАТЬСЯ В СЛОВАХ (putat'sja $v$ slovax (Prép) (litt. s'emmêler dans les mots) - «bafouiller »), nous retrouvons au contraire, le trait dépendance que subit $\mathrm{X}$ devant $\mathrm{Y}$. En effet, l'incompétence de $\mathrm{X}$ vient de cette privation de liberté causée par $\mathrm{Y}$. X se perd, se confond, bafouille... car il est dominé par les excuses, conjectures, mots...

83 Rappelons que ce sont souvent le sujet et l'objet prépositionnel qui déclenchent la lecture métaphorique. C'est peut-être pour cette raison que le rapport entre prépositions et métaphore suscite des opinions contraires. Ainsi, Anne-Marie Berthonneau ${ }^{24}$ écarte l'idée de l'existence d'emplois métaphoriques dans le domaine prépositionnel. Selon elle, l'emploi métaphorique, par exemple, temporel, d'une préposition spatiale n'éveille aucune impertinence catégorielle et semble stable et conventionnel.

En effet, on pourrait s'interroger, comment appliquer, un dispositif métaphorique aux prépositions qui, au lieu de correspondre à une classe d'entités dans le monde, sont le vecteur de l'expression d'une relation entre deux entités? Pour cela, nous disposons d'une description du sens des prépositions (configuration idéographique) qui permet de façon claire le transfert sélectif de certains traits du sens spatial de la préposition au sens temporel ou notionnel.

\subsubsection{Limitation et intériorisation}

Les traits limite et intérieur propres à la sémantique de la préposition /v/ la distinguent de celle de /na/ dans la relation positionnelle.

Prenons l'opposition GPrép. В доме (v dome (Prép.) - «dans la / une maison») vs. l'adverbe домА (doma - «à la maison, chez soi »). En français, le mot maison qui fait partie du domaine privé des personnes (de la sphère personnelle), est précédé de l'article défini qui suggère facilement la conscience de la relation étroite avec le sujet parlant ou avec la personne dont il s'agit. En russe, le degré de proximité entre le possesseur (locuteur / sujet) et l'objet possédé (la maison) est marqué uniquement dans la construction adverbiale et non pas dans la construction prépositionnelle.

87 Ainsi la préposition /v/ introduit une séparation distincte ou pose une limite entre l'observateur (le locuteur) et le repère (la maison) mais aussi entre le repéré (quelqu'un) et le repère : 
(13а) домА кто-то Есть.

Doma kto-to est'.

À la maison - quelqu'un (Nom.) - est.

Il y a quelqu'un à la maison.

[ = la maison du locuteur et/ou de la personne qui s'y trouve]

(13b) в ДОМЕ КТО-ТО ЕСТЬ.

V dome kto-to est'.

V - maison (Prép.) - quelqu'un (Nom.) - est.

Il y a quelqu'un dans la maison.

[ = la maison qui n'appartient pas forcément au locuteur ni à la personne qui s'y

trouve]

Dans le premier exemple, l'adverbe дома (doma) remplace le groupe prépositionnel в МоЁм дОмЕ ( $v$ mоёm dome (Prép.) - «dans ma maison»), alors que dans le second, la préposition / $/$ introduit un objet дом (dom - «la maison») considéré comme représentant d'une classe d'objets дома (doma - «les maisons ») et précise l'emplacement d'une tierce personne à l'intérieur de cet objet.

Mettons en contexte deux autres locutions - В ГЛАЗАХ (v glazax) et НА ГЛАЗАХ (na glazax) :

(14) У НЕЁ В ГЛАЗАХ МОЖНО ПРОЧИТАТЬ ВСЮ ЕЁ ЖИЗНЬ.

U neË v glazax možno pročitat' vsju eË žizn'.

Chez - elle (Gén.) - v - yeux (Prép.) - possible - lire (PF) - toute (Acc.) - sa - vie

(Acc.)

On pouvait lire tout son vécu dans ses yeux.

(15) ЭКОНОМИКА КИТАЯ РАЗВИВАЕТСЯ НЕ ПО ДНЯМ, А ПО ЧАСАМ НА ГЛАЗАХ У ВСЕГО МИРА.

Èkonomika Kitaja razvivaetsja ne po dnjam, a po časam na glazax u vsego mira.

Économie (Nom.) - Chine (Gén) - évolue (IPF) - non - par - jours (Dat.) - mais - par

- heures (Dat.) - na - yeux (Prép.) - chez - tout (Gén.) - monde (Gén.).

La situation économique de la Chine évolue à tire-d'aile, sous les yeux du monde entier.

La locution $\boldsymbol{v}$ glazax (dans les yeux) renvoie à un espace imaginaire qui intériorise des pensées reflétant ici le vécu d'une femme. Ainsi les yeux $(\mathrm{Y})$ contiennent des images et des idées formées sous l'influence du monde extérieur. La préposition /v/ renferme cet éventail imaginaire dans l'espace réduit des yeux qui reflètent habituellement l'âme humaine.

Contrairement à /v/, la préposition /na/ dans na glazax introduit métaphoriquement le champ de vision illimité se propageant au monde entier. L'action évoluer se manifeste dans un espace visible et ouvert, extérieur aux yeux de l'être humain. On ne parle plus du vécu intérieur réfléchi dans les yeux d'une personne mais du tableau extérieur exposé devant les yeux de chacun.

91 Après avoir évoqué ces quelques traits concernant la sémantique de la préposition /v/, nous nous proposons de dresser (exactement comme dans le cas de la préposition /na/) la configuration idéographique de /v/, suivie, toujours dans l'optique d'une synthèse, de sa classification d'après le trait dominant. 


\subsection{Bilan : configuration idéographique de la préposition / $/$}

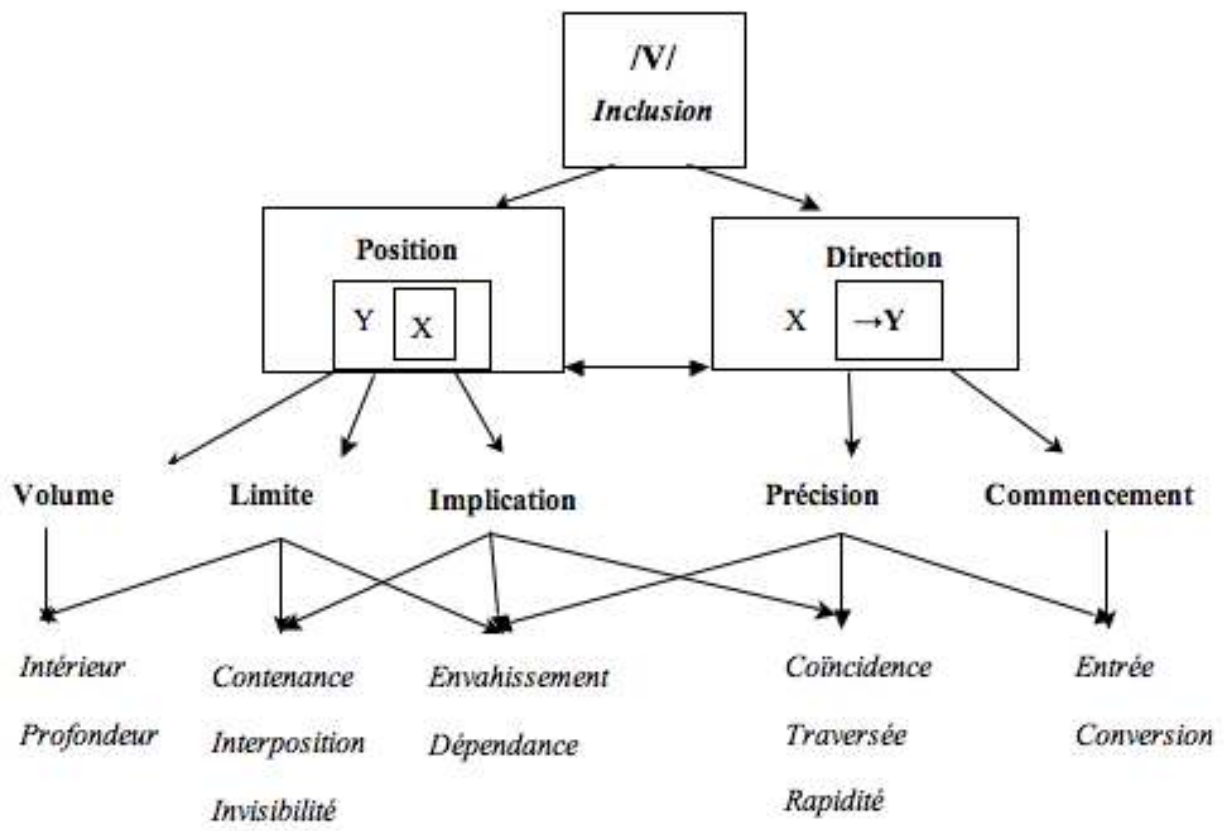

Classification d'après le trait dominant

1) VOLUME :

+ Activation du trait intérieur:

ИГРАТЬ ВО ДВОРЕ - jouer dans la cour igrat' vo dvore = jouer (IPF) - vo - cour (Prép.)

+ Activation du trait profondeur:

УТОНУТЬ В РЕКЕ - se noyer dans la rivière utonut' $\mathbf{v}$ reke $=$ se noyer $(\mathrm{PF})-\mathbf{v}$ - rivière (Prép.)

93 2) LIMITE :

+ Activation du trait contenance:

В ЕГО СЛОВАХ СЛЫШАЛАСЬ УГРОЗА - ses paroles étaient menaçantes

$\mathbf{v}$ ego slovax slyšalas' ugroza $=\mathbf{v}$ - ses - paroles (Prép.) - s'entendait (IPF) - menace (Nom.) ОСЕНнИЕ ЛИСТЬЯ В КУчЕ - les feuilles d'automne en tas osennie list'ja $\mathbf{v}$ kuče $=$ automnales - feuilles (Nom.) $-\mathbf{v}-$ tas (Prép.)

+ Activation du trait interposition:

ОНА ПРЯЧЕТСЯ В ПОДУШКАХ - elle se cache entre les coussins ona prjačetsja $\mathbf{v}$ poduškax $=$ elle - se cache (IPF) $-\mathbf{v}$ - coussins (Prép.)

+ Activation du trait invisibilité (recouvrement): РУКИ В ПЕРЧАТКАХ - les mains dans les gants ruki $\mathbf{v}$ perčatkax $=$ mains $-\mathbf{v}-$ gants (Prép.)

94 3) IMPLICATION :

+ Activation du trait dépendance:

Быть в долГАХ - avoir des dettes

byt' $\mathbf{v}$ dolgax = être (IPF) $-\mathbf{v}-$ dettes (Prép.) 
+ Activation du trait envahissement:

ОН весь В РАБоте - il est pris par le travail

ves' $\mathrm{v}$ rabote $=$ tout $-\mathrm{v}-$ travail (Prép.)

4) PRÉCISION :

+ Activation du trait coïncidence:

РОДИТЬСЯ в 1980 годУ - naître en 1980

ВЕРЁВКА ДЛИНОЙ В ДВА МЕТРА - la corde d'une longueur de deux mètres

+ Activation du trait traversée :

СТРЕЛЯТЬ В НОГИ - tirer dans les jambes

+ Activation du trait rapidité:

СДЕЛАТЬ В ОДИН ПРИёМ - faire d'un seul coup

sdelat' $\mathbf{v}$ odin priëm $=$ faire $(\mathrm{PF})-\mathbf{v}-$ un (Acc.) - procédé (Acc.)

5) COMMENCEMENT :

+ Activation du trait entrée:

ВМЕШАТЬСЯ В ССОРУ - intervenir dans la dispute

vmešat'sja v ssoru = se mêler (PF) $-\mathbf{v}$ - dispute (Acc.)

+ Activation du trait conversion:

ПРЕВРАТИТЬ ДОМ В РАЗВАЛИНЫ - transformer la maison en ruines.

prevratit' dom $\mathbf{v}$ razvaliny $=$ transformer $(\mathrm{PF})-$ maison $($ Acc. $)-\mathbf{v}-$ ruines (Acc.)

\section{Conclusion}

97 Nous avons avancé une hypothèse en supposant que la préposition pourrait se définir par de nombreux traits réunis dans une configuration idéographique qui ne sont pas nécessairement présents dans tous ses emplois, mais forment des combinaisons variables d'un emploi à l'autre. Ces traits n'ont pas la même saillance dans deux langues différentes, telles que le russe et le français.

98 Ainsi, à chacune des prépositions étudiées une signification unique abstraite a été attribuée (celle de superposition à /na/, celle d'inclusion à /v/), et différentes interprétations (ou relations), spatiales et non spatiales, ont été construites à partir des combinaisons de cette signification abstraite et des traits idéographiques dépendants avec certains éléments du contexte.

Ce concept relationnel dénotant une combinaison finie de traits a un caractère schématique. Le concept relationnel est envisagé comme le nœud d'accès au réseau de significations des prépositions.

Ainsi, la préposition /v/ met en relation d'inclusion non seulement les objets concrets mais aussi des entités abstraites lors des transferts métaphoriques, et la préposition /na/ ne marque pas uniquement la position « sur la surface » mais également et surtout ajoute le domaine d'une entité à un domaine de l'autre, en notant ainsi le voisinage séparé par une frontière des domaines notionnels mis en relation. 


\section{BIBLIOGRAPHIE}

Berthonneau A.-M. (1998), « Espace et temps : quelle place pour la métaphore ? », Verbum XX/4, pp. 353-382.

Franckel, J.-J. \& Paillard, D. (2007), Grammaire des prépositions, T. 1, Ophrys, Paris/Gap.

Franckel, J.-J. \& Paillard, D. (1998), « Aspects de la théorie d'Antoine Culioli », Langages 129, pp. 52-63.

Homma, Y. (2006), « Réflexions sur l'analyse de l'identité d'une préposition : le cas de dans », Modèles linguistiques. La préposition en français XXVII-2/54, Éditions des Dauphins, Toulon, pp. 25-36.

Korovko, L. A. (2004), Predlog On v anglijskom jazyke, referat, http://revolution.allbest.ru/ languages/

Langacker, R. W. (1991), Concept, Image, and Symbol: The Cognitive Basis of Grammar, Mouton de Gruyter, Berlin \& New York.

Leeman, D. (1997), « Sur la préposition en », Faits de langues, 9, Ophrys, Paris, pp. 135-143.

Leeman, D. (2008), « Prépositions du français : état des lieux », Langue française, 157, pp. 5-19.

Raxilina, E. V. (2000), Kognitivnyj analiz predmetnyx imen : semantika i sočetaemost', Russkie slovari, Moskva.

Sarda, L. (1999) Contribution à l'étude de la sémantique de l'espace et du temps : analyse des verbes de déplacement transitifs directs du français. Thèse de doctorat de l'Université de Toulouse le Mirail.

Selivërstova, O. N. (2000), « Semantièeskaja struktura predloga na », Issledovanija po semantike predlogov, Russkije slovari, Moskva, pp. 189-241.

SelivËrstova, O. N. (2004), Trudy po semantike, Jazyki slavjanskoj kul'tury, Moskva.

Selivërstova, O. N. \& Paillard D., Issledovanija po semantike predlogov, Russkie slovari, Moskva Svorou, S. (1994), The Grammar of Space, John Benjamins, Amsterdam.

Šanskij, N. M. (1971), Ètimologičeskij slovar' russkogo jazyka, Leningrad.

Talmy, L. (1978), « Figure and ground in complex sentences », Greenberg et al., Universals of human language, 4 , SUP, Stanford.

Tayar-Boulanger, A. (1985), Initiation à la Grammaire russe. Morphologie, Institut d'études slaves, Paris.

Ušakov, D. N. (1935-1940), Tolkovyj slovar' russkogo jazyka v 4-x tomax, Gosudarstvennyj institut «Soveckaja Ènciklopedija », Moskva.

Vandeloise, C. (1986), L'espace en français. Sémantique des prépositions spatiales, Éditions du Seuil, Paris.

Vandeloise, C. (1988). « Les usages spatiaux statiques de la préposition à », Cahiers de Lexicologie 53 (2) : pp. 119-148. 
Vandeloise, C. (1990), « Les frontières entre les prépositions sur et à », Cahiers de Grammaire 15, pp. 157-164.

Vandeloise, C. (1993), « Les analyses de la préposition dans : faits linguistiques et effets méthodologiques », Lexique 11, pp. 15-40.

Zolotova, G. A. (2006), Sintaksičeskij slovar'. Repertuar èlementarnyx edinic russkogo sintaksisa, izd. 3, Èditorial JRSS, Moskva.

\section{NOTES}

1. IPF : verbe imperfectif

2. Prép. : cas prépositif (prépositionnel)

3. Entité : chose réelle, existante, représentable par une image ou un concept, ensemble de moyens traduisant un réseau de relations de dépendance, n'existe que par l'association du signifiant et du signifié.

4. Leeman D. (1997), « Sur la préposition en », Faits de langues, 9, Ophrys, Paris, (135-143).

5. Le postulat fondamental du paradigme cognitiviste est que la structure sémantique des langues reflète nos modes de conceptualisation du monde (postulat défendu notamment par Ronald Langacker dans les années quatre-vingt). Il ne s'agit plus alors de saisir un fonctionnement d'un système posé comme autonome relativement à la réalité qui nous entoure ou à la manière dont nous nous la représentons, mais au contraire, à partir d'une hypothèse sur les mécanismes mentaux, d'établir un lien entre eux et le sens des unités linguistiques.

6. Franckel J.-J., Paillard D. (2007), Grammaire des prépositions, T. 1, Paris/Gap : Ophrys.

7. Le concept de repérage est central de la Théorie des opérations énonciatives d'Antoine Culioli. Il s'agit d'une opération langagière qui est le moteur de la détermination des différentes notions dans un énoncé, qui intervient dans la construction même de ces notions, dans la détermination nominale et verbale, dans les relations entre notions. Le terme repère est à son tour repéré par rapport à un autre terme repère. Ainsi, il n'existe pas de terme isolé, un terme, quel qu'il soit, ne pouvant acquérir une valeur référentielle que s'il est plongé dans un système de repérage.

8. Repéré : entité $\mathrm{X}$ localisée dans le temps et l'espace par rapport à une autre entité $\mathrm{Y}$ dans le cadre de la relation sémantique instaurée par une préposition.

9. Lakoff G. (1987), Women, fire, and dangerous things - What categories reveal about the mind, Chicago University Press, Chicago.

10. SelivËrstova, O. N. (2000), « Semantièeskaja struktura predloga na », Issledovanija po semantike predlogov, Russkije slovari, Moskva, pp. 189-241.

11. Compte tenu de la relation de repérage, nous avons préféré les termes repéré / repère à ceux de cible / site (employés par Claude Vandeloise pour désigner le localisé et le localisateur dans l'opération de localisation), ou à ceux de figure / ground (fond) (proposés par Léonard Talmy dans son modèle de la Dynamique des Forces (DF)).

12. Le terme idéographique est relatif à une écriture dans laquelle les idées et les objets sont représentés par des symboles (idéogrammes).

13. D'après Antoine Culioli (Théorie des opérations énonciatives), un terme ne renvoie pas à un sens, mais renvoie à un domaine notionnel, c'est-à-dire à tout un ensemble de virtualités. On construit un domaine notionnel en associant une notion à un mot. Ainsi, construit à partir d'une notion, le domaine notionnel permet de structurer la classe d'occurrences (ou l'extension de la notion) qui est associée à cette dernière.

14. KO rovko L. A. (2004), Predlog On $v$ anglijskom jazyke, referat, http://revolution.allbest.ru/ languages/

15. La préposition /na/, suivie du cas accusatif. 
16. Puisque le repéré est en mouvement, on préféra le terme mobile dans la configuration $\mathrm{X} / \mathrm{na} /$ $\mathrm{Y}$ (Acc).

17. A. Herskovits (1982) a noté la présupposition de mouvement qui existe pour les repérés introduits par la préposition à. Elle affirme que le contraste entre, par exemple, Le train est à la station Victoria et Le train est dans la station Victoria est dû au fait que la première phrase, mais non la seconde, évoque la trajectoire dont la position actuelle du train est le résultat.

18. En français, ces emplois de la préposition à ont été décrits par Claude Vandeloise comme des activités routinières, c'est-à-dire qu'à un lieu est associé une activité typique habituellement pratiquée dans ce lieu, si bien que l'expression typique de la localisation (être à) peut servir à décrire l'activité pratiquée dans ce lieu sans que la localisation soit finalement impliquée par la description. Par exemple, il est à l'Université peut certes signifier qu'il s'y trouve, mais peut aussi signifier qu'il y travaille (enseigne, étudie...), sans que la localisation soit présupposée par l'énoncé.

19. Šanskij N. M. (1971), Ètimologičeskij slovar' russkogo jazyka, Leningrad.

20. Fonction médiative: exprime le moyen de réalisation d'une action, vise à définir l'intervention d'un tiers $(\mathrm{Y})$ pour faciliter ou conditionner l'action de $\mathrm{X}$.

21. $P F$ : verbe perfectif

22. La variante de /v/, la préposition /vo/ (BO) est employée devant les mots commençant par une suite de consonnes, en particulier, si la première consonne est $B$ ou $\Phi$ (ВО ДВОРЕ (vo dvore "dans la cour »), во сколько (vo skol'ko - «à quelle heure »), во СНЕ (vo sne - «dans le sommeil »), Во ВЛАСТИ (vo vlasti - «dans le pouvoir »), ВО ВЗОРЕ (vo vzore - « dans le regard»), ВО ФРАНЦИИ (vo Francii " en France »), ВО РТУ (vo rtu - «dans la bouche »)), devant les mots déclinés BEСЬ (ves' - « tout ») et ВСЯкИЙ (vsjakij - «n'importe quel») (ВО ВЕСЬ ОПОР (vo ves' opor - « en toute vitesse »), ВО ВСЕХ МЕСТАХ (vo vsex mestax « dans tous les endroits »), ВО ВСЯКОМ СЛУЧАЕ (vo vsjakom slučae - " en tout cas »)), ainsi que dans les expressions littéraires devenues figées (Во-ПЕРВыХ (vo-pervyх - « en premier »), ВО ИмЯ (vo imja - « au nom de »), ВО ГЛАВЕ (vo glave - « en tête »)).

23. La préposition russe $3 A(/ \mathrm{za} /)$ a le sens spatial « derrière » (se traduit également par de l'autre côté de, au delà de, par delà : ЗА РЕКоЙ (za rekoj - «de l'autre côté de la rivière »)), le sens temporel « pendant » (ou durant, en, au cours de : ЗА ПОСЛЕДНИЕ ТРИ ГОДА (za poslednie tri goda - « au cours de trois dernières années $»)$ ), exprime la cause ou la conséquence (équivalant à pour, pour cause de: УВАЖАТЬ ЗА ХРАБРОСТЬ (uvažat' za xrabrost' - « estimer pour le courage »)), marque le but (se traduit par les infinitifs chercher, acheter, etc. : идите зА доктором (idite za doktorom - "allez chercher un médecin»)), exprime d'autres relations notionnelles correspondant à par, à, au lieu de, en guise de, en qualité de.

24. Berthonneau A.-M. (1998), «Espace et temps : quelle place pour la métaphore?», Verbum XX/4, pp. 353-382.

\section{RÉSUMÉS}

Dans le présent article, nous abordons la question de l'identité sémantique des prépositions russes $\mathrm{B}(/ \mathrm{v} /)$ et $\mathrm{HA}(/ \mathrm{na} /)$ en établissant pour chacune d'elles un schéma relationnel de base, en construisant leur configuration idéographique, en regroupant leurs relations sémantiques dans le but d'expliquer les emplois polysémiques de ces prépositions. Nous considérons la signification de la préposition comme un réseau complexe, où le sens abstrait de la préposition est précisé par 
un nombre d'éléments sémantiques (traits idéographiques et relationnels) qui font partie du contexte dans lequel se fixent les différents champs d'application de la préposition. Les systèmes prépositionnels $d u$ russe et $d u$ français ne sont pas identiques car les champs sémanticorelationnels des prépositions dans les deux langues sont différents : le caractère de la relation (l'activation des traits idéographiques) et le nombre d'entités mises en rapport, régies par telle ou telle préposition, diffèrent d'une langue à l'autre.

In the present article, we tackle the question of the semantic identity of the Russian prepositions « $B$ " and « $H A$ » by establishing for each of them a relational basic scheme, by building their ideographic configuration, by grouping together their semantic relations in order to explain the polysemous use of these prepositions. We consider that the meaning of the preposition is a complex system, where the abstract meaning of the preposition is specified by a number of semantic elements (ideographic and relational features) which are a part of the context in which settle the various fields of application of the preposition. The Russian and French prepositional systems are not identical because the semantico-relational fields of the prepositions are different in both languages: the character of the relation (activation of the ideographic features) and the number of entities in contact, governed by such or such preposition, differ from one language to another.

\section{INDEX}

Mots-clés : configuration idéographique, entité, préposition russe, relateur, trait relationnel, forme schématique de base

Keywords : entity, russian preposition, ideographic configuration, relational feature, relator, schematic basic form

\section{AUTEUR}

\section{TATSIANA VAVULA}

Université Paris-Sorbonne (Paris IV) 